\title{
Assessment of pain in herpes zoster: lessons learned from antiviral trials
}

Dworkin H.R ${ }^{[1]}$, Carrington $\mathrm{D}^{[2]}$, Cunningham A ${ }^{[3]}$, Kost R $\mathrm{G}^{[4]}$, Levin M J[5], McKendrick M $\mathrm{W}^{[6]}$, Oxman M N ${ }^{[7]}$, Rentier $\mathrm{B}^{[8]}$, Schmader K $\mathrm{E}^{[9]}$, Tappeiner $\mathrm{G}^{[10]}$, Wassilew $\mathrm{S} \mathrm{W}^{[11]}$, Whitley R J[12]

${ }^{[1]}$ Columbia-Presbyterian Medical Center, New York, NY, USA

${ }^{[2]}$ St George's Hospital, London, UK

${ }^{[3]}$ Department of Virology, Westmead Hospital, Westmead, Australia

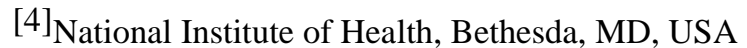

[5] University of Colorado Health Sciences Center, Denver, CO, USA

$\left.{ }^{[6}\right]_{\text {Royal Hallamshire Hospital, Sheffield, UK }}$

[7] University of California, San Diego, CA, USA

[8] University of Liege, Liege, Belgium

[9] Duke University Medical Center, Durham, NC, USA

[10] Universitatsklinik fur Dermatologie, Allgemeines Krankenhaus der Stadt Wien, Vienna, Austria

${ }^{[11]}$ Dermatologische Klinik, Klinikum Krefeld, Germany

${ }^{[12]}$ University of Alabama at Birmingham, Birmingham, AL, USA

\begin{abstract}
Pain typically accompanies acute herpes zoster and, in a proportion of patients, it persists well beyond rash healing. Pain must therefore be analyzed in trials of antiviral agents in herpes zoster, but different methods have been used to analyze pain in recent published trials. These reports are reviewed and their methodological strengths and weaknesses examined. Based on this review, recommendations for the design and analysis of future trials of antiviral agents in herpes zoster are proposed. The principal recommendation is that antiviral efficacy should be evaluated both by distinguishing post-herpetic neuralgia from acute pain and by considering pain as a continuum. The primary endpoint should address both the prevalence and duration of post-herpetic neuralgia and should be examined in those patients who have post-herpetic neuralgia. Adopting the proposed recommendations in design and analysis of future trials should facilitate comparison across trials of the efficacy of antiviral agents in the treatment of herpes zoster.
\end{abstract}

\section{Keywords}

Clinical trials; Herpes zoster; Pain analyses; Post-herpetic neuralgia; Primary endpoint; Zosterassociated pain

\section{Introduction}

Herpes zoster, or 'shingles', is a common disease, developing in as many as $20 \%$ of all people (Wood and Easterbrook, 1995). The disease occurs at any age, though its incidence rises with increasing age (Hope-Simpson, 1965).

World-wide, herpes zoster is associated with high morbidity, with pain representing the most troublesome aspect of the disease (Dworkin and Portenoy, in press). Most patients have pain prior to and during the rash. This acute phase pain, which tends to last for three to four weeks, is often described as excruciating. However, pain that persists long after the rash has disappeared, traditionally referred to as post-herpetic neuralgia (PHN), is of greatest concern. This prolonged pain does not respond well to analgesic therapy and can lead to behavioral complications, including depression. 
Indeed, PHN is by far the most common complication of herpes zoster (Hope-Simpson, 1965; Easterbrook and Wood, 1995).

As with the disease itself, the incidence of PHN also increases with age (de Moragas and Kierland, 1957; Guess et al., 1985; Harding et al., 1987). For example, PHN (defined as pain at 1 month or more) is relatively unusual in patients under 50 years, yet occurs in around $50 \%$ of patients aged 60 years (de Moragas and Kierland, 1957). Although there are several risk factors for PHN (Dworkin and Portenoy, in press), it is not presently possible to identify which patients with herpes zoster will develop PHN. Three oral antiviral agents are licensed for the treatment of herpes zoster: acyclovir, its prodrug valaciclovir, and famciclovir. In trials with these antivirals, pain has been an important efficacy parameter.

Wood and the Herpes Zoster Clinical Trial Consensus Group (1995) recently reported the results of a consensus meeting on herpes zoster pain that was held in July 1994. However, a number of seminal papers on the treatment of herpes zoster were not available at the time of this assessment (Degreef and the Famciclovir Herpes Zoster Clinical Study Group, 1994; Beutner et al., 1995; Tyring et al., 1995; Wood et al., 1996). A group of specialists therefore met to review the published data and assess the strengths and weaknesses of the various clinical trial designs and analyses. Based on this review, recommendations are proposed for the design and analysis of future trials of antiviral agents to treat herpes zoster. The emphasis of these recommendations is on the evaluation of long-term pain in herpes zoster. Ocular, visceral, and neurological complications in herpes zoster patients were considered important, but were beyond the scope of this meeting. Although the design and evaluation of clinical trials in patients with established PHN involves several of the issues that were discussed, these topics have been reviewed recently (Max et al., 1991; Watson, 1993; Watson and Chipman, 1993) and were also considered beyond the scope of the meeting.

\section{Methodology}

A group of 12 specialists in infectious diseases, dermatology, virology, geriatrics, and pain met in March 1996. Five papers which reported the results of antiviral trials of herpes zoster were reviewed by the participants (Degreef and the Famciclovir Herpes Zoster Clinical Study Group, 1994; Beutner et al., 1995; Tyring et al., 1995; Wood et al., 1994, 1996). The research designs, methods of data analysis, and results of these trials were systematically examined, in order to provide a basis for a comprehensive examination of the analysis of prolonged pain in herpes zoster trials.

\section{Pain in herpes zoster: issues and recommendations}

\subsection{Post-herpetic neuralgia or zoster-associated pain?}

Acute pain of herpes zoster has been described using the terms acute neuritis or acute herpetic neuralgia, and post-herpetic neuralgia (PHN) has been used to refer to pain persisting after a defined interval. These terms are generally acceptable but there are differing opinions on definitions, specifically about when one phase ends and the other begins. These opinions may depend on when, and by whom, the patient is evaluated. For those who see the patient shortly after the onset of the infection (e.g., general practitioners, infectious disease specialists, and dermatologists), acute pain is of most relevance and PHN is pain which persists beyond skin healing or for longer than 30 days from the onset of rash. However, when the patient is evaluated after the acute infection has resolved (e.g., by pain specialists and neurologists), PHN is typically considered as pain which persists for 3-6 months following the acute infection. Indeed, the reported incidences of PHN vary greatly depending on the definition (Fig. 1). Consequently, a new approach was introduced by Crooks et al. (1991) that considers herpes zoster pain as a continuum of zoster-associated pain (ZAP) from onset to resolution. In its most accurate sense, ZAP can encompass prodromal pain, acute pain, and PHN when this occurs in a patient, although in clinical trials, it is more appropriate to consider it as pain from enrollment in the study to complete resolution (Fig. 2). Although both PHN and ZAP can be used to assess the effects of antiviral agents on pain in clinical trials, some trials have reported analyses based on only one of these approaches; this makes comparison of the results of different trials difficult. Moreover, different analytic methods have been used to examine PHN. 


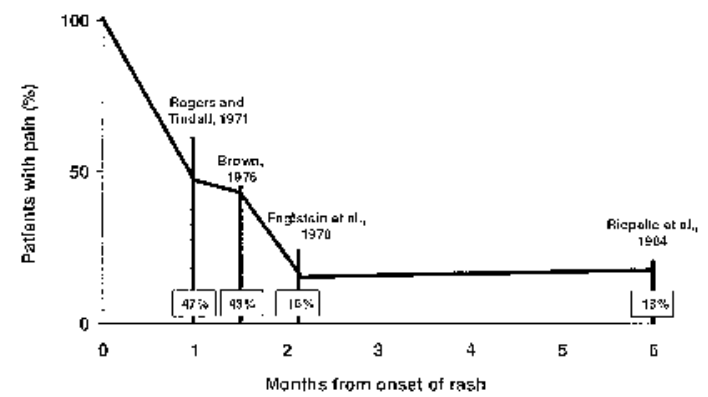

Fig. 1. Definitions of post-herpetic neuralgia (PHN) reported in the literature (\% incidence reported).

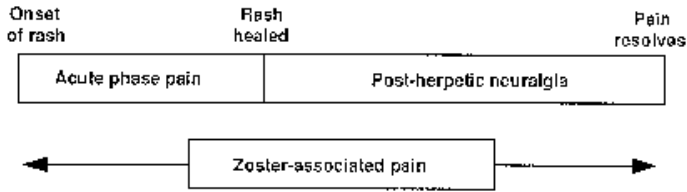

Fig. 2. Zoster-associated pain (ZAP), acute phase pain, and post-herpetic neuralgia (PHN).

\subsubsection{ZAP: strengths and weaknesses}

The pain associated with herpes zoster is generally experienced as a continuum from prodrome, through acute pain, to post-herpetic neuralgia, assuming that the pain does not resolve with the rash. Thus, ZAP is a meaningful measure from the patient's perspective, providing information on the duration of all pain.

A major advantage of ZAP is that it does not require any assumption concerning the start of PHN, as it makes no attempt to separate the two groups of patients, i.e. those with acute pain only and those who develop PHN.

However, the pathophysiology of acute pain and PHN are probably different (Bennett, 1994). In two studies using the McGill Pain Questionnaire (Melzack, 1975), the characteristics of acute phase pain and PHN were found to differ (Bhala et al., 1988; Bowsher, 1993). For instance, sharp or stabbing pains are more common in patients during the acute phase, whereas burning pain is more common in patients with PHN. In addition, at autopsy Watson et al. (1991) demonstrated dorsal horn atrophy in the sensory ganglion of the affected side in patients with PHN, but not in herpes zoster patients whose pain did not persist.

Because ZAP considers pain as a continuum (Fig. 2), it is difficult to assess whether the effect of an antiviral is on acute pain, on PHN, or on both. Since the majority of patients experience acute phase pain only, and fewer develop PHN, the duration of pain measured by ZAP will be heavily influenced by the resolution of acute pain. This effect is illustrated in Fig. 3, which shows data from placebotreated patients in a famci-clovir study (Dworkin et al., 1995a). The median time to loss of ZAP is very similar to the median time to loss of acute phase pain. However, when only those patients who have pain following healing are considered, the median and interquartile range for time to loss of pain from enrollment is much higher than for ZAP, as expected for a chronic pain condition.

As can be seen from these data, efficacy demonstrated using the concept of ZAP does not necessarily demonstrate efficacy with respect to PHN (according to the definitions of each), and the terms ZAP and PHN should not be used interchangeably since they do not measure the same thing. ZAP accurately depicts the prevalence of pain over time but provides no information on either the prevalence or the duration of PHN. It is also important to note that in evaluating the efficacy of antiviral agents, some regulatory authorities currently consider analyses of PHN prevalence or duration preferable to analyses of the ZAP continuum of pain (Feigal, 1995).

\subsubsection{PHN: strengths and weaknesses}

PHN has traditionally been used to describe prolonged pain in herpes zoster, which is a major clinical concern owing to its high prevalence in the elderly and its lack of response to analgesic intervention. 
The effects of antivirals on both the prevalence and duration of PHN can be assessed using a given definition of PHN.

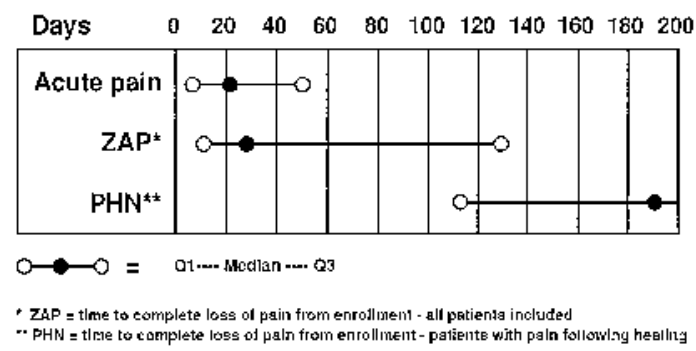

Fig. 3. Comparison of acute phase pain, zoster-associated pain (ZAP), and post-herpetic neuralgia $(P H N)$ in placebo-treated patients $(n=419)$.

To assess the effects of antiviral treatment given during the acute phase on the duration of PHN which develops subsequently in only some of the patients, it is necessary to exclude from the analysis those enrolled patients who do not develop PHN. Provided this exclusion is based on a prospective definition of PHN only (i.e., patients are not excluded for other reasons), then the analysis can be considered to be conducted on an intention-to-treat basis. However, because only some patients with herpes zoster develop PHN (and those that do cannot be identified at study entry), larger numbers of patients must be enrolled in a clinical trial in order to provide an adequate sample size of PHN patients for analysis. In order to examine only those patients with PHN, this group of patients must be defined. This reveals the major weakness of PHN, namely that it is not clear when acute phase pain progresses to PHN. Several definitions have been proposed (Fig. 1). Pain for greater than or equal to 30 days from enrollment is one definition that has been used. However, this does not adequately define PHN because some patients will still have rash at this time (Burgoon et al., 1957); thus, the pain reported by such patients may well be acute pain and not PHN.

Another definition is the presence of pain after healing of the rash. The main argument in favor of this definition is that healing is considered by some investigators to be an easily identifiable endpoint, after which the patient can be considered to be in the post-herpetic phase of the disease. Although there is a lack of a pathophysiologic basis for this definition, specifically that there is no evidence that rash healing is accompanied by the cessation of neural inflammation and acute neural pain, this definition does attempt to separate those patients with PHN from those with acute phase pain. It has been suggested that bias is introduced using this definition in antiviral trials because antiviral treatment may reduce time to healing (Wood and the Herpes Zoster Clinical Trial Consensus Group, 1995). However, antiviral therapy usually decreases healing time by only a few days (e.g., Wood et al., 1988; Tyring et al., 1995) whereas the duration of PHN can be reduced by two months (Tyring et al., 1995).

One method of overcoming this concern is to identify patients who have PHN (defined as those with pain following healing of the rash) and then calculate time to loss of pain from enrollment (Cunningham, 1995; Boon and Griffin, 1996), rather than from healing. This avoids any influence of differing rates of healing between two treatments and also excludes those patients who have only the shorter-lasting acute pain. Nevertheless, this approach to analyzing PHN neglects the potential contribution of the antiviral agent to the resolution of acute pain. Moreover, rash healing is a cutaneous event which may not be optimal for identifying those patients who have prolonged pain.

In order to distinguish clearly between those patients with PHN and those with acute phase pain, pain persisting at least 3 months from rash onset can be used as a specific (but less sensitive) definition of PHN. Use of this definition in clinical trials would ensure that the effects of antiviral agents on chronic pain would be evaluated. This definition is also consistent with the distinction between acute and chronic pain proposed in the International Association for the Study of Pain classification of chronic pain syndromes (Merskey and Bogduk, 1994).

An analysis of PHN requires that a subgroup of patients be examined because not all patients experience prolonged pain. Hence, this approach has been criticized as not fulfilling intention-to-treat principles. The effect of an antiviral on PHN, however, can be evaluated only by examining those patients who have PHN according to a prospectively defined criterion. Nevertheless, it is important to ascertain that the groups of patients with PHN who are studied in such an analysis are comparable with respect to demographic features and relevant covariates.

An alternative method has been employed to include all patients enrolled. Using this method, patients who do not have PHN are assigned a duration of PHN of zero days (Beutner et al., 1995; Wood et al., 
1996). However, the number of such patients included in different treatment groups may not be balanced. The effect of including patients who do not develop PHN and designating them as having a time to event of zero days is illustrated in Fig. 4, which is based on placebo-treated patients from a large herpes zoster study.

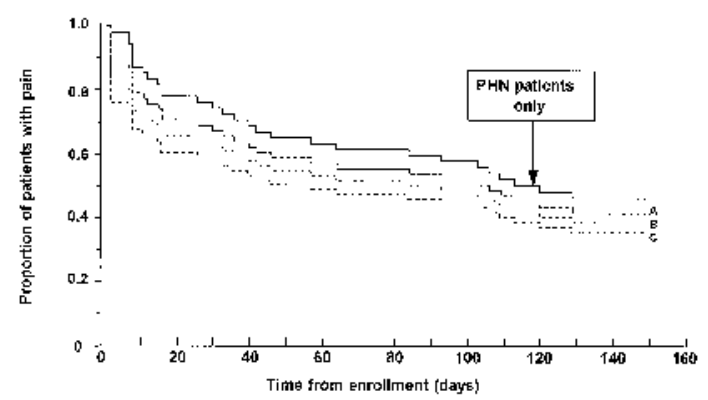

Fig. 4. Effect of adding patients without PHN into a PHN analysis (model data). Control population: PHN patients only (i.e., those patients with PHN defined as pain following healing of the rash), $n=228$. A: Control population $(n=228)$ plus $8 \%(n=20)$ patients without PHN (assigned as 0 days to event). B: Control population $(n=228)$ plus $15 \%(n=40)$ patients without $P H N$ (assigned as 0 days to event). $C$ : Control population $(n=228)$ plus $26 \%(n=80)$ patients without PHN (assigned as 0 days to event).

\begin{tabular}{|c|c|c|c|c|}
\hline & $\begin{array}{l}\text { Contr } \\
\text { ol }\end{array}$ & $\begin{array}{l}\text { A vs. } \\
\text { Control }\end{array}$ & $\begin{array}{l}\text { B vs. } \\
\text { Control }\end{array}$ & $\begin{array}{l}\text { C vs. } \\
\text { Control }\end{array}$ \\
\hline $\begin{array}{l}\text { No. of } \\
\text { patients }\end{array}$ & 228 & 248 & 268 & 308 \\
\hline $\begin{array}{l}\text { No. } \\
\text { without }\end{array}$ & 0 & 20 & 40 & 80 \\
\hline $\begin{array}{l}\text { PHN } \\
\text { included } \\
\text { in analysis }\end{array}$ & $(0 \%)$ & $(8 \%)$ & $(15 \%)$ & $(26 \%)$ \\
\hline $\begin{array}{l}\text { Median } \\
\text { duration } \\
\text { (days) }\end{array}$ & 119 & 108 & 92 & 41 \\
\hline $\begin{array}{l}\text { Hazard } \\
\text { ratio }^{\mathrm{a}}\end{array}$ & - & 1.15 & 1.28 & 1.51 \\
\hline $95 \%$ CIs & - & $0.91-1.46$ & $\begin{array}{l}1.02- \\
1.62\end{array}$ & $1.22-1.89$ \\
\hline$P$ value & - & 0.25 & 0.03 & 0.002 \\
\hline
\end{tabular}

As the percentage of patients who do not have PHN (those assigned a value of zero days) increases, so does the difference between the groups. This results in an increase in the hazard ratio and a decrease in the $P$-value when the Cox Proportional Hazards model is used to analyze the data. It is also clear that the inclusion of patients who do not develop PHN results in an 'artificially' lowered median duration of PHN. Moreover, if one antiviral agent influences the resolution of acute events more than another, a bias will be introduced if such events are used in this approach to determine which patients have PHN.

\subsubsection{Consensus: PHN or ZAP?}

Both ZAP and PHN are of value in assessing the efficacy of antiviral agents in the treatment of herpes zoster. ZAP reflects the patient's experience of the total pain of herpes zoster but, as it does not permit antiviral efficacy to be assessed with respect to the prolonged pain of PHN, the target endpoint for antiviral efficacy assessment should be PHN.

With respect to PHN analyses, patients experiencing clinically significant pain beyond 3 months from 
enrollment should be included. This would account for patients whose pain fluctuates over this period. As discussed below, only those patients with moderate or greater severity pain should be included in these analyses because the persistence of mild pain is unlikely to be clinically significant.

\subsection{Intention-to-treat and efficacy-evaluable analyses}

Most published clinical trials of antiviral agents report results based on the intention-to-treat sample. However, some also report the findings of the efficacy-evaluable group (Degreef and the Famciclovir Herpes Zoster Clinical Study Group, 1994). The value of analyses of the efficacy-evaluable group has been a controversial issue in herpes zoster trials, as in most clinical trials. Differences may be statistically significant in the efficacy-evaluable group but not in the intention-to-treat sample. When this occurs, it may be because patients who are unlikely to receive benefit from a drug have been included in the intention-to-treat analysis. In such instances, the efficacy-evaluable analysis may yield a more accurate assessment of the potential beneficial effects of the drug. It can be expected, however, that the direction of differences in efficacy between treatments should be the same for both intentionto-treat and efficacy-evaluable analyses.

\subsubsection{Consensus: intention-to-treat or efficacy-evaluable analyses?}

Intention-to-treat analysis reflects more closely the real situation outside of the clinical trials setting and is required by regulatory authorities for approval purposes. However, including patients who do not have the condition (e.g., patients proven to have zosteriform herpes simplex infections) or who are less likely to derive benefit from therapy because of non-compliance with study medication makes it difficult to evaluate the true effects of an antiviral agent. Hence, efficacy-evaluable analyses are valuable in determining whether such agents can have beneficial effects in herpes zoster patients. Efficacy-evaluable analyses could exclude, for example, non-compliant patients and protocol violations, with all criteria for exclusion prospectively defined. Similarly, the intention-to-treat sample should also be prospectively defined (e.g., all patients randomized, all patients receiving at least one dose of a study medication, or all patients meeting the entrance criteria).

\subsection{Should adjustments be made for covariates?}

Adjusting for factors that have been shown to have an influence on the primary endpoint has become a feature of most recent trials. It is generally recognized that adjusting for co-variates in this manner can have a profound effect on hazard ratios and confidence intervals. Consequently, it is important to consider whether all potential covariates should be included in adjusted analyses of antiviral efficacy, whether only those covariates that are considered to be associated with the endpoint should be included, or whether only those prospectively defined covariates that significantly affect the endpoint should be included. This question is important because the greater the number of covariates to be included in the analyses, the larger the sample size must be to ensure that the effect of each covariate can be determined accurately.

Covariate analyses are valuable for examining factors that predict prolonged pain and better therapeutic response; for pivotal trials, however, such analyses should be few in number and prospectively identified, with adjustments made for multiple comparisons if necessary. Older age and greater pain severity at enrollment have been found to be associated with prolonged herpes zoster pain in a large number of studies (e.g., McKendrick et al., 1994; Boon and Griffin, 1995; Beutner et al., 1995; Dworkin et al., 1995b, 1996; Whitley et al., 1996; Wood et al., 1996). Because of these robust associations, these two factors should be examined in all studies of herpes zoster and PHN.

\subsubsection{Consensus: adjust for covariates?}

Adjustments should routinely be made for the pre-specified covariates of age and pain severity at enrollment. Further, adjustments for six other covariates (Dworkin and Portenoy, in press) should be made if there is evidence that the factor has some effect $(P \leq 0.2)$ on the particular endpoint being evaluated in logistic or Cox regression analyses. These covariates are sex, race, rash severity at enrollment (proportion of the dermatome affected or number of lesions), rash duration at enrollment, 
presence and duration of prodrome, and site (ophthalmic versus other). Other covariates which could be examined in exploratory analyses, depending on whether they have been assessed in a trial, are sensory deficit in the affected and contralateral dermatomes, co-morbidity, and psychosocial factors.

\subsection{Is there a value in looking at subgroups?}

There are several arguments in favor of including subgroup analyses in herpes zoster trials. The main ones include: if the pre-specified sub-grouping factor makes the subgroup more sensitive to the event (e.g., increased age), then the power of analyses of that group to detect differences becomes greater; viewing the subgroup analyses in conjunction with the analyses of the whole population will provide a more complete picture of antiviral efficacy.

Arguments against including subgroup analyses include: patients may no longer be comparable in terms of baseline characteristics of prognostic significance; if a large number of comparisons are made between subgroups, the risk of statistical Type I false-positive error increases; the number of patients in an analysis is reduced.

\subsubsection{Consensus: subgroups}

Some of the reviewed trials enrolled patients aged 18 years or more and then analyzed those aged 50 years or more as a separate subgroup (Tyring et al., 1995; Wood et al., 1996). This was considered acceptable provided the subgroups were pre-specified and could be shown to be balanced with respect to the important co-variates. However, since PHN is rare in patients under the age of 50 years, future clinical trials to assess antiviral efficacy on pain in immuno-competent patients should include only those of 50 years of age or more. A subgroup analysis of patients aged 65 years or more can then be recommended. Since the incidence of PHN increases with advancing age, this subgroup analysis could provide additional efficacy data.

The more severe the pain is at enrollment, the longer the duration of pain tends to be (e.g., Dworkin et al., 1995b, 1996; Whitley et al., 1996). Therefore, a subgroup analysis of patients with moderate or greater pain severity at enrollment is recommended. Similarly, prodromal pain may influence the duration of prolonged pain (Beutner et al., 1995), and patients with prodromal pain for either less than 3 days, 3 days or more, or none at all, would be worthwhile subgroups to analyze.

Furthermore, there is evidence that earlier therapy with some antivirals may result in faster resolution of pain (Degreef and the Famciclovir Herpes Zoster Clinical Study Group, 1994). Subgroup analyses of patients for whom treatment was initiated within $24 \mathrm{~h}, 24-48 \mathrm{~h}$, or 48-72 h of rash onset may also reveal differences in efficacy between drugs.

\section{Designing the ideal zoster trial}

Based on the review of published trials and the discussion and recommendations presented above, other elements of clinical trial design were considered. A summary of the discussion and the recommendations that emerged from it are presented below.

\subsection{Which patients should be included in and excluded from a study?}

Trials to date have generally enrolled either patients of 18 years or more, or only patients over the age of 50 years. Since pain is relatively rare in patients aged less than 50 years, future herpes zoster trials in immunocompetent patients should include only patients aged 50 years or more when long-term pain is the major endpoint.

Occasionally, patients have been excluded from data analyses in antiviral trials when they did not report pain at enrollment (Huff et al., 1993). However, patients with little or no pain soon after rash onset may go on to develop prolonged pain (Huff et al., 1993) and should therefore be included in antiviral trials.

Although some trials have enrolled patients with ophthalmic zoster, the number of patients included, even in the larger studies, are insufficient to determine effects on pain using a subgroup analysis. Also, the primary reason for treating these patients was to prevent ocular complications and not necessarily to avoid or reduce acute or chronic pain, and certainly insufficient numbers will be enrolled to determine 
effects on ocular complications. The disease course in patients with ophthalmic zoster may differ from the course in patients with other distributions (Harding et al., 1987). It is also difficult to determine whether PHN in this group of patients is the same as in herpes zoster affecting other dermatomes, because lesions within the eye can persist for much longer than cutaneous lesions. Furthermore, ophthalmologists often use a topical antiviral in addition to systemic medication, and so many of these patients would be excluded in any event. For these reasons, patients with ophthalmic zoster should not be included in trials of uncomplicated herpes zoster. Separate trials should be undertaken to study this more complex condition.

Other exclusion criteria are disseminated disease, and patients taking immunosuppressive medications, narcotic analgesics for pre-existing pain conditions, or antivirals with activity against varicella-zoster virus, including topical antivirals.

Most trials have excluded patients whose rash has been present for more than $72 \mathrm{~h}$, as it has been argued that there is little benefit in treating these patients. Not only does viral replication start in nerves during the prodromal phase (before the rash appears), but also one study has revealed that treatment of patients within $48 \mathrm{~h}$ of rash onset may result in a faster rate of resolution of ZAP (Degreef and the Famciclovir Herpes Zoster Clinical Study Group, 1994). However, antiviral efficacy in patients enrolled beyond $72 \mathrm{~h}$ of rash onset has not been adequately studied for acy-clovir therapy, and not studied at all for the newer agents famciclovir and valaciclovir. Antiviral therapy may be of benefit to the patient even if the rash is beyond $72 \mathrm{~h}$ of onset if new lesions are still forming at this point. A separate study for those enrolled between 73 and $144 \mathrm{~h}$ of rash onset could be considered and would be worth undertaking to evaluate the efficacy of antiviral treatment initiated more than 3 days after rash onset when there is evidence of continuing viral replication.

Although patients using topical antivirals should be excluded, it is unclear whether or not to exclude other topical preparations, such as lidocaine and calamine, which could interfere with pain assessments. Other possible exclusion criteria include antidepressants and patients with pre-existing chronic pain syndromes, who would probably be taking analgesics on a regular basis, which again could interfere with pain measurements. However, too many restrictions would be unwise, as patient recruitment into studies would become very difficult.

\subsection{What should the power be?}

Future herpes zoster trials should ideally be conducted with $90 \%$ power, although this is not mandatory. A lower power may be problematic, particularly if only a single trial is being conducted. Demonstrating equivalence of two drugs is more difficult than demonstrating superiority of one over the other, and larger numbers of patients are needed to do so. Despite this, equivalence studies have the advantage that either equivalence or superiority can be demonstrated in one study. In contrast, superiority studies can demonstrate whether or not one drug is superior to another but equivalence cannot be concluded if superiority is not detected.

In multicenter studies, an adequate number of patients at each center must be included. Centers enrolling one or two patients can be a source of bias.

\subsection{How frequently should patients be assessed?}

The reviewed clinical trial publications reveal wide variation in the frequency of assessment. A balance must be reached, therefore, between sufficient assessments to determine the endpoint accurately, but not so many assessments as to make a trial impossible for patients.

For duration of PHN, the possibility of following patients for one year can be considered; however, a 6month follow-up is much more feasible. A nested cohort design with follow-up to 12 months could be employed for patients with pain at the 6-month assessment (although patients with intermittent pain could be missed). The reason for this nested cohort design is the limited number of patients with PHN at 6 months. In assessments of the prevalence of PHN, some patients will be lost to follow-up, potentially causing an imbalance between treatment groups. For assessments of the duration of PHN, however, this would not be a problem as patients lost to follow-up would be 'censored'.

Since the main reason for treating patients with herpes zoster is to prevent PHN or reduce its duration, assessment frequency should be designed to meet these requirements. Therefore, pain assessments are recommended twice in the first week (days 0 and 3/4), weekly until 1 month (days 7, 14, 21, and 28), and subsequently biweekly to the end of a trial. Pain assessments should be performed in the physician's office or at the patient's home (by a visiting nurse or physician) during the first month. 
Subsequently, this 'face-to-face' approach should be continued where possible; if this is not possible, telephone assessments can be used. However, if the effect of an antiviral on cutaneous healing is to be determined, then more frequent assessments during the first 2-3 weeks may be required.

Telephone assessments may not be a desirable method, partly due to the problem in finding people at home, but also to cultural differences. For example, patients in some countries do not like to be contacted in this way. Patient diaries are also an option. However, these are subject to recall biases as patients may forget or neglect to complete them at the required time and then fill them in after the fact or before the 'due date' (Friedman et al., 1982). Diaries could, however, be supplemented with prompting telephone calls around the time that the diary needs to be completed. It is important to recognize that many factors, including the environment and the time of the day, affect patients' perceptions of pain, and such considerations should play a role in the design of antiviral trials.

\subsection{What should be assessed?}

\subsubsection{Severity of pain}

In evaluating the presence of pain, previous herpes zoster trials have generally included any report of pain, even mild pain. However, there is a possibility that mild pain may be solicited by questioning and may not be indicative of PHN. Therefore, it can be recommended that only moderate to severe pain be considered as PHN; such pain is more likely to interfere with patients' functioning and have an adverse effect on quality of life (Lydick et al., 1995).

Four methodologies for measuring pain severity were discussed: visual analog scales, numerical rating scales, verbal rating scales, and the McGill Pain Questionnaire (Melzack, 1975). Visual analog scales can be difficult for elderly patients to use. The choice among the remaining three types of pain measures should be at the discretion of the investigator. Numerical rating scales, in particular a scale from 0-10 (ranging from no pain to the worst pain imaginable) have a number of desirable features and can be recommended for use in many situations. Verbal rating scales and the McGill Pain Questionnaire (Melzack, 1975) can be problematic in that they may need to be translated for use in some countries; in addition, the latter, which was designed as an interview, can be time consuming. However, many different language versions of the McGill Pain Questionnaire exist, and a shorter version is available (Melzack, 1987). Assessment of pain severity should include pain experienced over the last $24 \mathrm{~h}$, plus the worst pain since the last assessment.

\subsubsection{Type of pain}

The consensus was that five distinct types of pain are associated with PHN. These are steady throbbing pain, steady burning pain, intermittent sharp or shooting pain, allodynia (either burning or sharp/shooting pain in response to a stimulus that does not normally provoke pain), and hyper-pathia (an abnormally painful reaction to a stimulus, especially a repetitive stimulus, as well as an increased threshold; Merskey and Bogduk, 1994). Interestingly, burning pain appears to be less common in PHN when an antiviral has been administered (Bowsher, 1993). Investigations of the different qualities of pain should be exploratory. Information on the type of pain could be collected either with specific questions designed to capture this information or by using the McGill Pain Questionnaire.

\subsubsection{Burden of illness}

A method for quantifying pain in herpes zoster has been proposed in which the total burden of pain is examined (Oxman et al., 1994). This approach can be used in examining the continuum of pain from enrollment to resolution, or in applying the distinction between acute phase pain and PHN. Pain severity (e.g., on a 0-10 scale) is plotted against duration, and the resulting measure of burden of pain is the total area under the curve. The total area under the curve could be divided at day 30 or at the 3month point to give separate measures for acute phase pain and PHN.

This method is, as yet, untested but its inclusion in an upcoming trial will reveal its utility.

\subsubsection{Quality of life and health care utilization}


The impact of disease on quality of life has been examined in recent antiviral trials (Beutner et al., 1995; Whitley et al., 1996), and could be readily examined in future trials. Quality of life parameters include physical disability, role and occupational disability, social disability, psychological distress (e.g., depression and somatic preoccupation), and sleep disturbance. Elderly patients do not fear pain any more than younger patients; rather, what is often feared is the effect that the pain has on independence and enjoyment of life. Health care utilization is becoming increasingly important and this should be assessed prospectively in herpes zoster trials. However, the impact of PHN in the general population is likely to be very different from that in the setting of a clinical trial.

\subsubsection{Analgesic use}

In antiviral trials, there should be a limited list of analgesic and antidepressant drugs from which physicians or general practitioners could choose in treating persisting pain. However, this approach would be more difficult in multinational studies and in cases where the investigator needed to prescribe specific analgesia. It is less clear whether analgesic use should be examined simply as a secondary endpoint or whether it should be accounted for in quantifying pain. Although the latter would seem to be an important refinement in assessing zoster pain, arguments against it include the fact that analgesics can be taken either continuously or intermittently (as pain recurs) and that assumptions would need to be made regarding analgesic effectiveness. At a minimum, all analgesic use should be routinely recorded so that such analyses could be conducted.

\section{Recommendations for future zoster trials}

In light of the above considerations, the following recommendations are proposed.

\subsection{Primary endpoint}

The primary endpoint in herpes zoster trials should assess PHN defined as clinically significant pain of a duration equal to or greater than 3 months from enrollment.

Clinically significant pain should be considered as pain of at least moderate severity. One approach to defining such pain is that pain reported to be three or greater on a 0-10 numerical rating scale. This criterion is based on the results of Lydick et al. (1995), in which ratings of acute pain in herpes zoster patients of one or two on a $0-10$ scale were rarely accompanied by adverse impacts on quality of life. Two analyses should be conducted: PHN prevalence, assessed as point prevalence of clinically significant pain at three months, and PHN duration, assessed as duration of clinically significant pain in those patients with PHN. Patients should be assessed for 6 months following enrollment regardless of any pain-free interval, and a nested protocol should then be conducted to examine PHN for an additional 6 months.

The use of this primary endpoint will mean, of course, that the number of patients to be enrolled will have to be greater than the number enrolled in most antiviral trials in herpes zoster conducted to date. In a recent meta-analysis of acyclovir trials, at 3 months after rash onset $25 \%$ of patients 50 years of age and above had pain (Wood et al., 1996). In a trial designed to demonstrate the superiority of a new antiviral agent compared with acyclovir using this endpoint, for $80 \%$ power to demonstrate a $40 \%$ reduction in pain at the $P<.05$ level, 250 patients would be required in each arm; for $90 \%$ power, 335 patients would be required in each arm. For the sake of illustration, if it is assumed that one-half of these patients have mild pain, then for $80 \%$ and $90 \%$ power these sample sizes would need to be increased to 565 and 750 patients per arm respectively to demonstrate a $40 \%$ reduction in the prevalence of pain of at least moderate severity. Although these are large sample sizes, antiviral trials approaching this size have been conducted in herpes zoster patients (Beutner et al., 1995) and will be necessary to evaluate the effects of new antiviral treatments on the development of clinically significant post-herpetic neuralgia.

\subsection{Secondary endpoints}

The recommended secondary endpoints are: duration of clinically significant ZAP, defined as above; duration of ZAP of any pain severity; duration and prevalence of PHN defined as pain of any severity of a duration equal to or greater than 3 months from enrollment; duration and severity of acute pain (in 
patients who only have acute pain); pain interference with activities of daily living; health-related quality of life; and analgesic use. In addition, a combined pain/analgesic score could also be explored as a secondary endpoint.

\subsection{Other study design elements}

Other recommendations for future zoster trials in immunocompetent patients are as follows:

(1) Inclusion criteria should be patients of 50 years of age or above enrolled within $72 \mathrm{~h}$ of rash onset.

(2) Exclusion criteria should be patients with ophthalmic zoster or disseminated disease, and patients on immunomodifying medication, narcotic analgesics for pre-existing pain conditions, or antivirals with varicella-zoster virus activity.

(3) An intention-to-treat analysis should be performed routinely, but the efficacy-evaluable group should also be examined to determine antiviral efficacy. Both analyses need to be prospectively defined.

(4) Adjustments should be made for age and pain severity at enrollment. Adjustment for other covariates, such as sex, race, rash severity at enrollment, rash duration at enrollment, presence and duration of prodrome, and site, should be made only if sufficient evidence is found that these covariates affect the endpoint.

(5) The preferred power of a study should be $90 \%$, though this is not mandatory, and equivalence studies may be preferable.

(6) Subgroup (exploratory) analyses could include: patients 65 years of age or older; patients with moderate or greater pain severity at enrollment; patients for whom treatment was initiated within 24, 48 , or 48-72 $\mathrm{h}$ of rash onset; and patients with no prodromal pain and prodromal pain for less than $72 \mathrm{~h}$ and for $72 \mathrm{~h}$ or more.

(7) Pain assessments should be done twice in the first week, weekly until one month, and then biweekly. These should be conducted in the physician's office or patient's home during the first month. (8) Pain severity should be measured using either numerical rating scales, verbal rating scales, or the McGill Pain Questionnaire. However, numerical rating scales are preferred.

(9) Pain severity should take into account pain experienced over the preceding $24 \mathrm{~h}$, plus the worst pain since the last assessment. For analyses of the duration of PHN, any patient experiencing clinically significant pain at 3 months or more from enrollment should be included.

(10) Investigations into the different qualities of pain should be conducted on an exploratory basis in future clinical trials.

(11) Quality of life parameters that should be considered include: physical disability, role/ occupational disability, social disability, psychological distress, and sleep disturbance.

(12) There should be a limited list of analgesic drugs from which physicians or general practitioners could choose.

\section{Conclusions}

Based on a review of existing trials, recommendations are proposed for evaluating pain in future herpes zoster trials. These recommendations have not been simultaneously applied in any one herpes zoster trial conducted to date. Hopefully, future trials by academia, government, and industry will do so. This will increase the comparability of data collected in antiviral trials, and thereby facilitate evaluations of the relative efficacy of different antiviral agents.

\section{Acknowledgements}

The authors are indebted to Simon Bishop, Ron Boon, David Griffin, Robin Saltzman, and Diane Davies for their invaluable assistance in the preparation of this article, and to SmithKline Beecham Pharmaceuticals for supporting the meeting on which this article is based.

\section{References}

Bennett, G.J. (1994) Hypotheses on the pathogenesis of herpes zoster-associated pain. Ann. Neurol. 35 Suppl., 38-41. 
Beutner, K.R., Friedman, D.J., Forszpaniak, C., Andersen, P.L. and Wood, M.J. (1995) Valaciclovir compared with acyclovir for improved therapy for herpes zoster in im-munocompetent adults. Antimicrob. Agents Chemother. 39, 1546-1553.

Bhala, B.B., Ramamoorthy, C., Bowsher, D. and Yelnoorker, K.N. (1988) Shingles and postherpetic neuralgia. Clin. J. Pain 4, 169-174.

Boon, R.J. and Griffin, D.R.J. (1995) Efficacy of famciclovir in the treatment of herpes zoster: Reduction of pain associated with herpes zoster. Neurology 45 Suppl. 8, 76-77.

Boon, R.J. and Griffin, D.R.J. (1996) Famciclovir: efficacy in zoster and issues in the assessment of pain. In: J. Mills, P.A. Volberding and L. Corey (Eds.), Antiviral Chemotherapy 4: New Directions for Clinical Application and Research, pp. 17-31. Plenum Press, New York.

Bowsher, D. (1993) Sensory change in postherpetic neuralgia. In: C.P.N. Watson (Ed.), Herpes Zoster and Postherpetic Neuralgia, pp. 97-107. Elsevier, Amsterdam.

Brown, G.R. (1976) Herpes zoster: correlation of age, sex, distribution, neuralgia and associated disorders. South. Med. J. 69, 576-578.

Burgoon, C.F., Burgoon, J.S. and Baldridge, C.D. (1957) The natural history of herpes zoster. JAMA $164,265-269$.

Crooks, R.J., Jones, D.A. and Fiddian, A.P. (1991) Zoster-associated pain: an overview of clinical trials with acyclovir. Scand. J. Infect. Dis. Suppl. 80, 62-68.

Cunningham, A.L. (1995) Clinical success in treating patients with famciclovir: optimism for the future. Res. Clin. Forums 17, 45-53.

de Moragas, J.M. and Kierland, R.R. (1957) The outcome of patients with herpes zoster. AMA Arch. Dermatol. 75, 193-196.

Degreef, H. and the Famciclovir Herpes Zoster Clinical Study Group (1994) Famciclovir, a new oral antiherpes drug: results of the first controlled clinical study demonstrating its efficacy and safety in the treatment of uncomplicated herpes zoster in immunocompetent patients. Int. J. Antimicrob. Agents 4, $241-246$.

Dworkin, R.H., Boon, R.J. and Griffin, D.R.J. (1995a) Famci-clovir: effect on pain in herpes zoster. Antiviral Res. 26, 344.

Dworkin, R.H., Boon, R.J. and Griffin, D.R.G. (1995b) Co-variates in herpes zoster and interpretation of clinical trial data. Antiviral Res. 26, 344.

Dworkin, R.H., Cooper, E.M., Walther, R.R. and Sweeney, E.W. (1996) Predicting the development of postherpetic neuralgia: a prospective study of herpes zoster patients. 8th World Congr. Pain, Vancouver, Canada, August 1996.

Dworkin, R.H. and Portenoy, R.K. (In press) Pain and its persistence in herpes zoster. Pain. Eaglstein, W.H., Weinstein, G.D. and Brown, J.A. (1970) The effects of early corticosteroid therapy on the skin eruption and pain of herpes zoster. JAMA 101, 132-139.

Easterbrook, P. and Wood, M.J. (1995) Post-herpetic neuralgia: what do drugs really do? In: S.L. Sacks, S.E. Straus, R.S. Whitley and P.D. Griffiths (Eds.), Clinical Management of Herpes Viruses, pp. 211-235. IOS, Amsterdam.

Feigal, D.W. (1995) Summary basis of approval for NDA 20-487 (valaciclovir caplets). Department of Health and Human Services, Washington, D.C.

Friedman, L.M., Furberg, C.D. and DeMets, D.L. (1982) Fundamentals of Clinical Trials, p. 135. PSG, 
London

Guess, H.A., Broughton, D.D., Melton, L.J. III and Kurland, L.T. (1985) Epidemiology of herpes zoster in children and adolescents: a population-based study. Pediatrics 76, 512- 517.

Harding, S.P., Lipton, J.R. and Wells, J.C.D. (1987) Natural history of herpes zoster ophthalmicus: predictors of posther-petic neuralgia and ocular involvement. Br. J. Opthalmol. 71, 353-358.

Hope-Simpson, R.E. (1965) The nature of herpes zoster: a long-term study and a new hypothesis. Proc. R. Soc. Med. 58, 9-20.

Huff, J.C., Drucker, J.L., Clemmer, A., Laskin, O.L., Connor, J.D., Bryson, Y.J. and Balfour, H.H. Jr. (1993) Effect of oral acyclovir on pain resolution in herpes zoster: a reanal-ysis. J. Med. Virol. Suppl. $1,93-96$.

Lydick, E., Epstein, R.S., Himmelberger, D. and White, C.J. (1995) Herpes zoster and quality of life: a self limited disease with severe impact. Neurology 45 Suppl. 8, 52-53.

Max, M.B., Portenoy, R.K. and Laska, E.M. (1991) The Design of Analgesic Trials, pp. 729. Raven Press, New York.

McKendrick, M.W., Care, C.D., Ogan, P. and Wood, M.J. (1994) A retrospective study of the epidemiology of zoster with particular reference to factors pertinent to the development of chronic pain. 2nd Int. Conf. Varicella-Zoster Virus, Paris.

Melzack, R. (1975) The McGill Pain Questionnaire: major properties and scoring methods. Pain 1, 275-299.

Melzack, R. (1987) The short-form McGill Pain Questionnaire. Pain 30, 191-197.

Merskey, H. and Bogduk, N. (Eds.), (1994) Classification of Chronic Pain: Descriptions of Chronic Pain Syndromes and Definitions of Pain Terms (2nd ed.). IASP Press, Seattle, WA.

Oxman, M.N., Levin, M., Johnson, G.R., Arbeit, R., Barry, P., Gershon, A.A., Schmader, K., Straus, S.E., White, C.J., Collins, D. and Colling, C.L. (1994) Trial of varicella vaccine for the prevention of herpes zoster and its complications (VA cooperative study no. 403): Vol. 1: protocol. Infectious Diseases Section, Veterans Affairs Medical Center, San Diego, CA.

Riopelle, J.M., Naraghi, M. and Grush, K.P. (1984) Chronic neuralgia incidence following local anesthetic therapy for herpes zoster. Arch. Dermatol. 120, 747-750.

Rogers, R.S. III and Tindall, J.P. (1971) Geriatric herpes zoster. J. Am. Geriatr. Soc. 19, 495-504.

Tyring, S., Barbarash, R.A., Nahlik, J.E., Cunningham, A., Marley, J., Heng, M., Jones, T., Rea, T., Boon, R., Saltz-man, R. and the Collaborative Famciclovir Herpes Zoster Study Group (1995) Famciclovir for the treatment of acute herpes zoster: effects on acute disease and postherpetic neuralgia: a randomized, double-blind, placebo-controlled trial. Ann. Intern Med. 123, 89-96.

Watson, C.P.N. (1993) Herpes Zoster and Postherpetic Neuralgia, pp. 258. Elsevier, Amsterdam. Watson, C.P.N. and Chipman, M. (1993) Suggestions for research and unanswered questions regarding postherpetic neuralgia. In: C.P.N. Watson (Ed.), Herpes Zoster and Postherpetic Neuralgia, pp. 239253. Elsevier, Amsterdam.

Watson, C.P.N., Deck, J.H., Morshead, C., Van der Kooy, D. and Evans, R.J. (1991) Post-herpetic neuralgia: further post-mortem studies of cases with and without pain. Pain 44, 105-117.

Whitley, R.J., Weiss, H., Gnann, J.W. Jr., Tyring, S., Mertz, G.J., Pappas, P.G., Schluepner, C.J., Hayden, F., Wolf, J., Soong, S. and the National Institute of Allergy and Infectious Diseases Collaborative Antiviral Study Group (1996) Acyclovir with and without prednisone for the treatment of herpes zoster: a randomized, placebo-controlled trial. Ann. Intern. Med. 125, 376-383. 
Wood, M.J., Ogan, P.K., McKendrick, M.W., Care, C.D., McGill, J.I. and Webb, E.M. (1988) Efficacy of oral acy-clovir treatment of acute herpes zoster. Am. J. Med. 85 Suppl. 2A, 79-83.

Wood, M.J. and Easterbrook, P. (1995) Shingles, scourge of the elderly: the acute illness. In: S.L. Sacks, S.E. Straus, R.S. Whitley, and P.D. Griffiths (Eds.), Clinical Management of Herpes Viruses, pp. 193-210. IOS, Amsterdam.

Wood, M.J. and the Herpes Zoster Clinical Trial Consensus Group (1995) For debate: how should zoster trials be conducted? J. Antimicrob. Chemother. 36, 1089-1101.

Wood, M.J., Johnson, R.W., McKendrick, M.W., Taylor, J., Mandal, B.K. and Crooks, J. (1994) A randomized trial of acyclovir for 7 days or 21 days with and without pred-nisolone for treatment of acute herpes zoster. N. Engl. J. Med. 330, 896-900.

Wood, M.J., Kay, R., Dworkin, R.H., Soong, S.-J. and Whitley, R.J. (1996) Oral acyclovir therapy accelerates pain resolution in patients with herpes zoster: a meta-analysis of placebo-controlled trials. Clin. Infect. Dis., 22, 341-347. 